\title{
Benefits of a New Approach to Designing Milling Cutter Using Metal Additive Manufacturing
}

\author{
Pavel Hanzl, Ivana Zetková \\ Faculty of Mechanical Engineering, University of West Bohemia. Univerzitní 2732/8, 30614 Pilsen. Czech Republic. \\ E-mail: hanzlp@rti.zcu.cz, zetkova@rti.zcu.cz
}

\begin{abstract}
Topological optimization is the process of reducing part weight while respecting strength requirements. This paper focuses on its possible positive consequences for the machining process. The main aim is to carry out a survey to obtain knowledge that will be applied during the topological optimization of a milling tool. According to all the indicators, the efficient implementation of lattice structures into the milling concept has the potential to achieve a high level of innovation, since the functional weight reduction of the tool allows for higher dynamics of the cutting process. The modified rigidity of the milling cutter and vibration absorption can extend the life of the cutting edge, and such a milling tool would provide a competitive advantage on the tool market.
\end{abstract}

Keywords: Milling tool, Topological optimization, Direct Melting Laser Sintering, Metal Additive Manufacturing

\section{Introduction}

The machining process is highly unstable and changes over time. Vibrations are excited by fluctuation of the cutting forces, changing friction conditions and varying stiffness. Whether it is a self-excited vibration or excited oscillation, the vibration has a negative impact on the shape accuracy of the machine surface, the tool life and the machine service interval. [17]

Oscillation can never be suppressed completely in a machine-tool-workpiece system, but proper adjustment of this system can restrict undesirable consequences which would affect the quality of the cutting process. To achieve maximum efficiency of the cutting process, all of the system components must be at the limits of their usable capacity. This state is not usually achieved in practice and the full potential of the machine-tool-workpiece system is not reached.

This article focuses on increasing the usable potential of a cutting tool, a face mill. The basic premise is the ability to withstand cutting forces. Typical approaches to tool rigidity are based on weight gain which leads to a decrease in tool dynamics, which requires a more powerful spindle drive, which is more expensive and less energetically favourable. Therefore, the tool price is not the only factor in total costs.

Modern approaches go the other way, trying to reduce tool weight and increase efficiency using available materials and design modifications. In professional practice, the process of reducing weight with respect to the functionality of the component is termed 'topological optimization' and one of its methods is lightening the component by the implementation of a lattice structure. If the optimization is done correctly, the resulting product shows an unusual combination of properties, such as high strength and rigidity accompanied by relatively low weight. Good oscillation absorption may be included in this. [3]

Therefore, the main aim of this article is to conduct a survey that focuses on the possible benefits of porous structures for a lightweight face mill. Applications are also sought where a milling cutter optimized using lattice structures will be most visible. For the production of an advanced milling cutter, it is necessary to use a relatively new production method, Metal Additive Manufacturing (MAM). This production method is currently a very hot topic, because it is one of the main elements of Industry 4.0. MAM can produce highly complex parts such as an optimized face mill by using lattice structures. Melting metal material using DMLS technology is competitive with other conventional methods such as forming. [2]

A number of hypotheses have arisen from the survey. One is the potential ability of a lattice structure to dampen shocks. An experiment is carried out to investigate this. The damping properties of the porous body are compared with a solid body using modal analysis. Another question is how the natural frequencies of the cutting tool change after implementing lattice structures. These and other hypotheses are dealt with in this article.

\section{Aspects Affecting the Cutting Process}

Face milling is a productive way of machining material. There are many aspects which may be affected by implementing lattice structures into the body of a face mill. The biggest changes are in the weight and stiffness of the cutting tool.

Cutting force, as a manifestation of the cutting process, is a basic prerequisite for machining. However, the machining process is unstable due to the fluctuation of the cutting force, etc. This means that the mill is subjected to dynamic loading. Since acceleration and movement have a considerable impact on the strength of the material.

One of the most important relationships for dealing with dynamic tasks is d'Alembert's principle, which is based on Newton's Law on the Motion of a Particle. It states that the sum of all forces acting on a body in the direction of oscillation, including inertial effects and damping effects, is equal to zero (see Fig.1). 

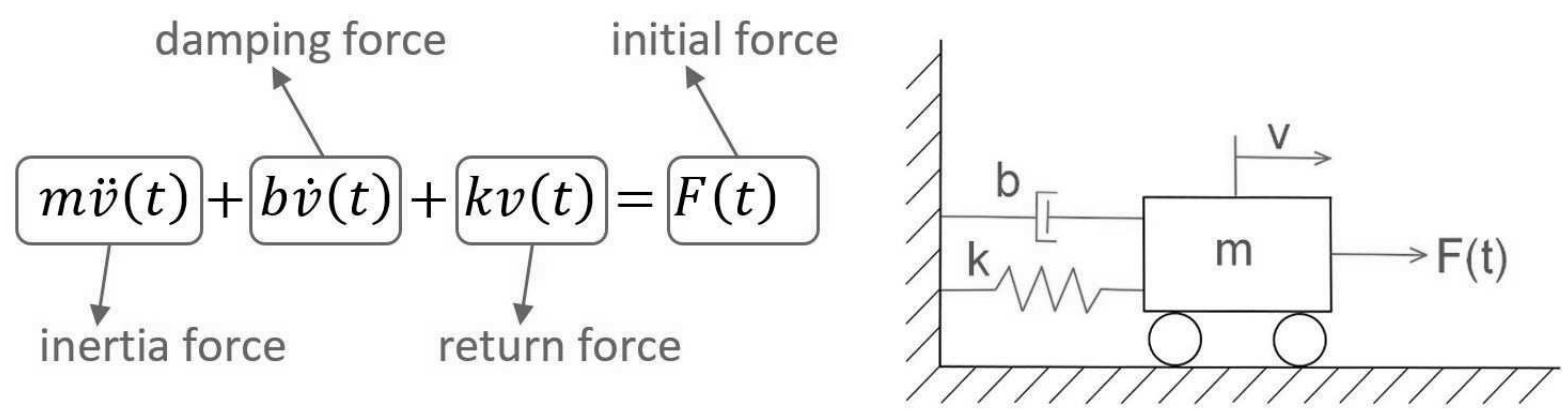

Fig. 1 Equation of d'Alembert's principle in solving dynamic action

Centrifugal effects increase in importance as the cutting diameter increases. This is due to the distribution of the rotating mass relative to the axis of rotation. The mass distribution expresses the established physical magnitude, the moment of inertia ' $\mathbf{J}$ ' (see Formula 1). This moment increases as the mass is further away from the centre of rotation, which also carries a high kinetic energy during rotation around the axis (see Formula 2). Therefore, the mass and proper centring affects the kinematic energy of the mill body.

$$
\begin{gathered}
\mathrm{J}=\sum_{k=0}^{n} m_{k} \cdot R_{k}{ }^{2} \\
\boldsymbol{W}_{\boldsymbol{k i n}}=\frac{\mathbf{1}}{\mathbf{2}} \boldsymbol{J} \cdot \boldsymbol{\omega}^{\mathbf{2}}
\end{gathered}
$$

$m_{k} \ldots \ldots \ldots$ weight of mass point

$R_{k}$........the perpendicular distance of the mass point from the rotation axis

$\omega$ angular speed

Traditional, unbalanced, heavy milling cutters with larger diameters cause vibrations, machine stress and poorly machined surfaces. Lightweight cutters improve high speed milling by reducing the vibration level and machine spindle power requirements while maintaining sufficient stiffness. Higher tool kinetics is also allowed. [4]

The cutting edge repeatedly penetrates the machined workpiece and leaves the cutting area at the angle of engagement. This cyclical stress exposes the tool repeatedly to considerable shocks, but modern cutting materials tolerate this kind of stress well due to their high compressive strength. The combination of optimized mill stiffness with damping properties has a considerable potential for the progress of cutting edge durability. This was confirmed by Miyaguchi, et al. [5], who showed that a ball end mill with an optimal tool length shows significant improvement and a longer tool life can be achieved by reducing tool stiffness, because the cutting forces are balanced.

The pioneering works by Wallace, Andrew [6] and Peters, et al. [7] identify the damping process as energy dissipation due to the interference between the cutting tool relief, clearance and the workpiece. Shock damping and vibration absorption is considered to be beneficial in machining. [8]

The vibration level is commonly determined by modal analysis, which is one of the most widely used methods for determining the frequency and shape of the system oscillations. The outputs describe characteristics such as natural frequencies and the damping properties of the system.

Calvalho et al. [9] focused on the natural frequency as a parameter affecting power consumption. They confirmed that if a tool operates in the vicinity of its natural frequency, the cutting forces increase and the energy consumption increases. By reducing vibration, the energy efficiency increases, the quality of the machined surface improves and the possibility of increased productivity opens up due to the option of selecting more aggressive cutting conditions.

However, increasing the feed rate or cutting speed results in increased stress on the cutting tool. Optimizing stiffness with shock absorption can be an effective approach to dealing with higher tool stress generated on the cutting wedge. A porous core system can be seen as a system of spring elements which are individual cell struts. These reduce the stiffness of the mill and limit the peak of the maximum load generated on the cutting edge. In this case, there is a risk, as when driving a car on an uneven surface without shock absorbers, that the machining process may become unstable. Therefore, it may be appropriate to include damping elements in the lightweight milling cutter assembly to stabilize the cutting process. These elements include, for example, filling the free space between the struts of the porous core using some form of damping material such as resin, rubber or even a cooling fluid, which would provide damping and absorb thermal energy at the same time.

\section{Choice of lattice structure topology}

Lattice structures can be considered as a solid phase with cavities whose mechanical properties depend primarily on the properties of the material which they are made from, and on the topology defining their geometry. Possibilities for the production of topologically optimized components with porous structures are limited if there are high demands on material properties, and here Metal Additive Manufacturing technology is almost the only production option.

There are several issues associated with the production of porous structures using MAM. Technologies such as SLM or DMLS are based on the principle of laser melting a metal powder layer by layer. Therefore, it cannot melt a layer for which the lower layer does not provide sufficient support. If this condition is not met, the resulting product will not match the model template and it will 
contain defects and its functionality may be affected. Therefore, the chosen porous topology has to be adapted to MAM and the expected loads during the cutting process.

Most regular lattice structures built using MAM are composed of straight struts. These structures are similar to bridge constructions (truss structures) or scaffolding. But the scale of lattice structures is much smaller. The basic cell for MAM is commonly in the range of 2-10 $\mathrm{mm}$. Maxwell developed a stiffness theory of truss structures and provided a necessary condition for maximum stiffness.

The struts of lattice structures can deform by either bending or stretching. In practice, most lattice structures are bending-dominated. Lattice structures that are strength-dominated are more weight-efficient in the task of material saving. There are topological criteria that determine the deformation mechanism from the analysis of struts and number of nodes. [10]
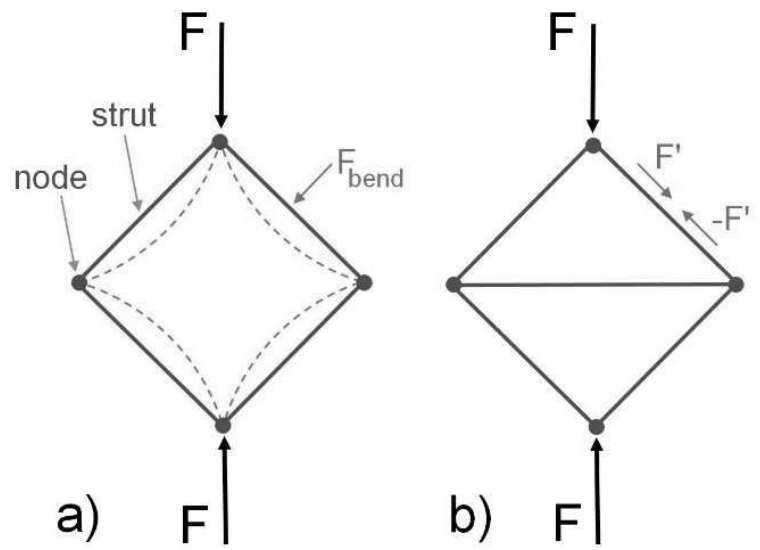

Fig. 2 Strut morphology a) bend-dominated b) strengthdominated

The following figure shows a simplified model of the effect of strut count on the force. The morphology in Figure 2a lacks rigidity. By adding one strut, a triangular morphology is created, which decomposes the external force to axial loading of the struts. In the first case, the load causes bending moment in the struts. Macroscopic rigidity or kind of the stress in the struts do not affected by avoiding rotation in the nodes. Even when the struts are bent, tension/pressure loads still prevail in the struts. Therefore, the load capacity of the morphology is mainly determined by the axial strength of the struts since the stretch-dominated topology has a higher load capacity relative to the weight. [10]

Deshpande, et al. [10] analysed the optimal count of struts per node. This count was determined from an extended Maxwell equation for three-dimensional strut structures (3) that takes into account stress states and mechanisms. The fulfilment of this criterion is a necessary condition for strength-dominated lattice topology. If a lattice structure has to resist only tensile load effectively, the minimum strut count is 6 connected in a node. The maximum count for all loading states (complex combined load) is established as 12 struts per node. More struts would lead to overestimation and narrowing of the strut diameter if weight is a priority.

b....count of struts

$$
b-3 j+6=s-m
$$

$j$.....count of node with frictionless connection s.....states of self-stress

m...mechanisms

Axial strain in the struts may be less favourable for damping characteristics due to buckling stress of the struts. In the case of damping, bending-dominated topologies may be preferred, because they respond more sensitively to load deflection as the generated deflection can be better absorbed by the damping component (such as rubber) contained in the core of the lattice topology. However, this assumption cannot be verified or refuted. No sources have been found on this topic.
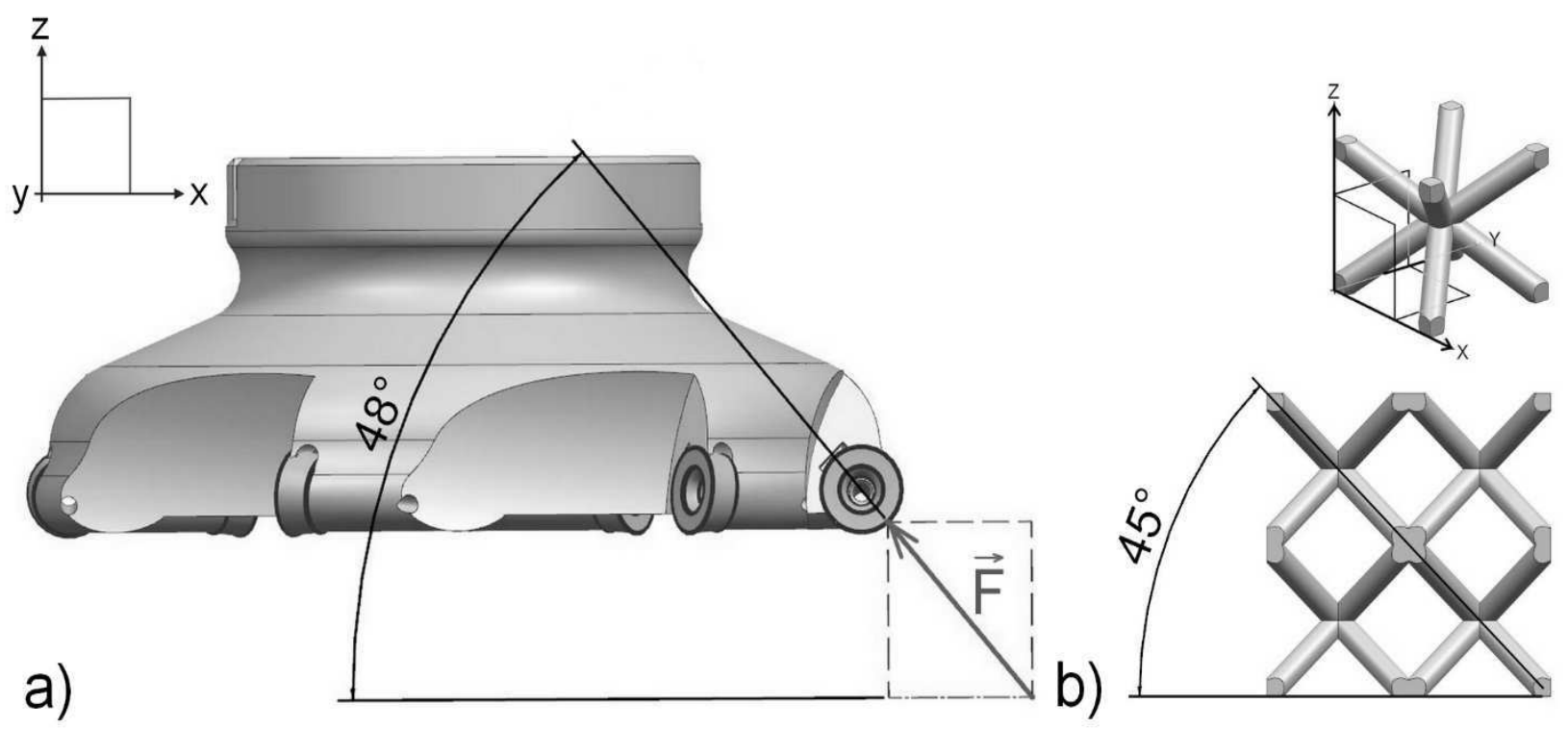

Fig. 3 a) Vector of axial cutting force b) Orientation of struts in BCC porous core with trimetric view on the base cell of BCC topology 
In view of these circumstances, the $\mathrm{BCC}$ topology was chosen for the input experiments. It meets the manufacturing criteria of MAM with the selected orientation and has 8 struts per node. Taking into account that the optimized milling cutter will have a cutting diameter of $125 \mathrm{~mm}$ and will use circle mill inserts, then the cutting force vector is approximately at an angle of $48^{\circ}$ in the axial plane of the face mill. The force vector was measured on a reference conventional mill with the same cutting diameters and 6 circle inserts (see Figure 3a). The cutting conditions are maintained for the other experiments.

When implementing BCC topology into a mill body while preserving geometry, the struts are loaded almost along their axis, thus maintaining the most suitable orientation for MAM production (i.e. building direction is along the $\underline{z}$ axis). The $45^{\circ}$ vector associated with the orientation of the BCC structure is shown in Figure $3 \mathrm{~b}$. This orientation could be advantageous in the task of finding maximum load and experiments will be conducted on this topic.

\section{Dynamic response of BCC topology}

Lattice structures are composed of a system of struts, which can be seen as spring elements. The BCC topology belongs to the category of strength-dominated structures if it is stressed by a uniaxial load. This follows from Maxwell's rule. Combined stresses from two types of load can also be absorbed well. This kind of stress is likely to be dominant in the porous core of a mill. The study [11] states that lattice structures have good energy absorption characteristics. This property is very welcome because the face milling cutting process is accompanied by interruptions caused by vibration and shocks.

The question therefore arises whether the BCC topol-

ogy exhibits damping properties. An experiment was performed to find the answer. A shell of a sample was filled with a BCC structure. The porous core was $30 \mathrm{~mm} \times 15$ $\mathrm{mm}$ and the basic cell size was $5 \mathrm{~mm}$ with $13.5 \%$ volume fraction. The thickness of the shell was set at $1 \mathrm{~mm}$ of volume material. Figure 5a shows the printed sample with a cross section of the model, and the real assembly of the experiment is shown in Figure 5b.

a)

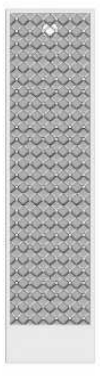

b)

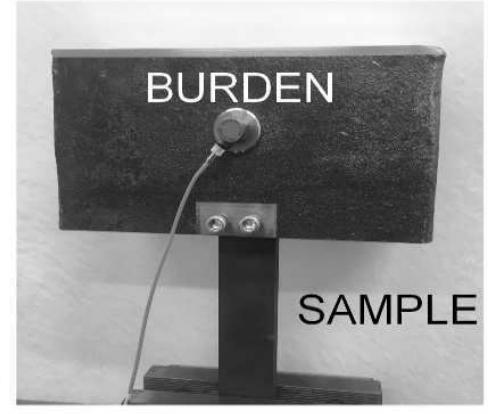

Fig. 5 a) porous sample b) setting of damping experiment

The difference in the weight between the solid sample and the porous sample is $0.66 \mathrm{~kg}$ and $0.28 \mathrm{~kg}$. The mass of the burden is much heavier, $12.95 \mathrm{~kg}$, to neglect the weights of the samples. Equipment from Brüel \& Kjaer was used to measure the oscillation: an accelerometer 4533-B-001, impact hammer 8206-003, and electronic module 3160-A-042. Frequency range of this measuring assembly is $0.2 \mathrm{~Hz}$ to $12.8 \mathrm{kHz}$ and resonance frequency of the accelerometer was determined to $38.7 \mathrm{kHz}$ which is more than sufficient for the experiment. The tested assembly was clamped in the vice of a rigid milling machine. The measured dynamic responses are shown in Figure 5.

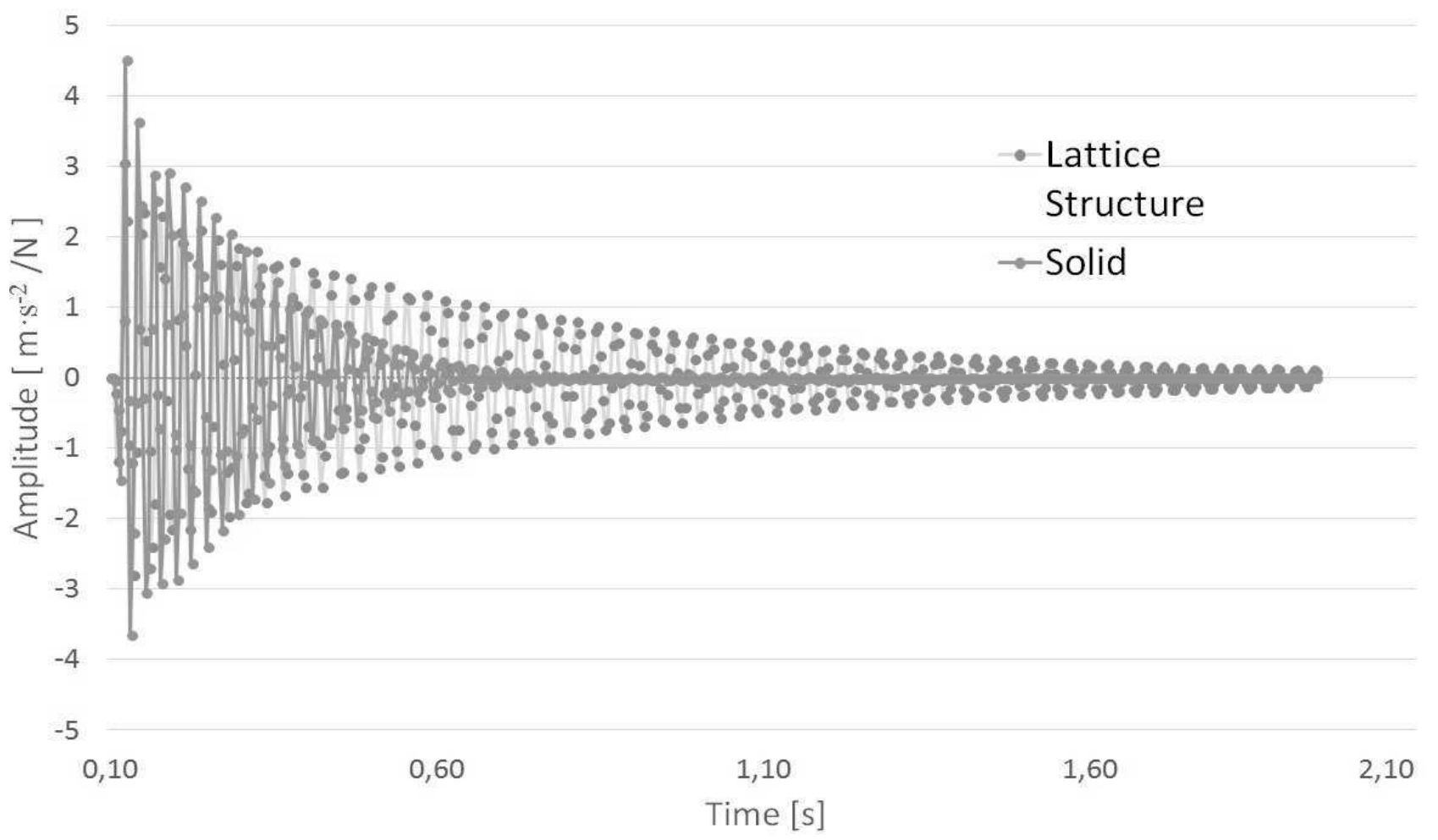

Fig. 5 Oscillation of Lattice Structure and Solid 


\section{Results and discussion}

This paper investigates and clarifies the damping behaviour of lattice structures. Analysis of the oscillations proved that lattice structures have worse damping properties than solid materials. The response is described by the exponential decaying equation (4) [12], which is the envelope of the oscillation, where the damping coefficient $\gamma$ [13] (or also the exponential decay [14]) for the sample with a lattice structure was 1.7 and the solid sample was 5.3. The results show that the porous sample had more than 3 times worse damping properties than the solid sample. This means that the porous specimen needs a longer time to reach a stabilized state without oscillation.

$$
A(t)=A_{o} \cdot e^{-\gamma t}
$$

Ao...peak amplitude of oscillation

e.... Euler's number

$\gamma$.....exponential decay

t....time

Damping properties may be improved by filling the free volume of the lattice structure with viscoelastic material [1]. This was confirmed by [15] when the amplitude of the specimen was effectively reduced without significantly shifting the natural frequency. However, insertion of viscoelastic material into the core of the lattice structure increases the mass and contradicts one of the greatest advantage of implementing structures.

\section{Conclusion}

The main aim of this paper was to carry out an extensive survey into how valuable lattice structures can be for milling tools. These lightweight and firm structures are pivotal because they will form the core of new mill designs and will affect the resulting behaviour during the cutting process.

General trends in productive machining are towards reducing vibration and increasing the tool dynamics, which leads to better stability and efficiency of the cutting process. Some practical advice for how to reduce vibration is given below. [16]

- Use the lightest cutter body possible

- Use a round insert cutter/balance the radial and axial cutting forces

- Use a milling cutter with internal cooling channels for proper supply

Lattice structures are a particularly interesting solution for achieving maximum weight reduction. Effective production will result from the expanded use of Metal Additive Manufacturing on the market. However, there are some rules which must be followed to achieve successful production of an optimized milling cutter by using lattice structures. The development of a new generation of milling cutter has three components: manufacturability, adaptation to stress conditions, and added value of the complete design solution.
According to the primary indicators, $\mathrm{BCC}$ topology is interesting for the cutter, as it conforms to Maxwell's rule where the minimum strut per node of lattice structure is six for uniaxial stress. BCC topology provides eight struts per node, which could be sufficient for the purposes of a milling cutter. The axes of the struts are very close to the expected cutting force vector. The BCC unit is also suitable in terms of manufacturability. The building direction of its struts enables reliable production using Metal Additive Manufacturing.

However, the damping properties of this structure are very limited and significant improvements cannot be expected by using other lattice structures, although the damping effects can be enhanced by filling the free volume of the structure with rubber, etc. This is another direction that research can take to improve mill performance. The damping test pointed out that lattice structures are not good vibration absorbers but could be good isolators of vibration, provided that the strength of the porous structure does not isolate vibration transferred to the system, or rather, attenuates the amplitude of oscillation. [3]

Therefore, it can be expected a reduction of some qualitative characteristics of the lightweight cutter such as stiffness and natural frequency. The decreasing these properties does not necessarily mean a worse resultant machined surface. Because there is a large reserve and milling cutter with porous core can provide higher dynamics of machining process due to the reduction of weight.

There are further benefits from using MAM, such as the possibility of integrating a complex system of cooler channels into the body of the milling cutter. The direction of further research in this area is based on this knowledge. To achieve the high efficiency of a topologically optimized milling cutter and conserve its structural integrity, a trade-off has to be made between stiffness and weight. In total, the lightening of a milling cutter has the potential to bring a high degree of innovation.

\section{Acknowledgement}

This paper was supported by the Internal Grant Agency of the University of West Bohemia, project No. SGS2016-005.

\section{References}

[1] BAKOŠOVÁ, D. (2018) Dynamic Mechanical Analysis of Rubber Mixtures filled by Carbon Nanotubes, Published by Manufacturing Technology, ISSN 1213-2489, June 2018, Vol. 18, No.3, Pp. 345-351.

[2] KUČEROVÁ, L., ZETKOVÁ, I. (2016) Metallography of 3D Printed 1.2709 Tool Steel, Published by Manufacturing Technology, ISSN 1213-2489, February 2016, Vol. 16, No. 1, Pp. 140-144.

[3] SYAM, W.P., JIANWEI, W., ZHAO, B., MASKERY, I., ELMADIH, W., LEACH, R. (2018) Design and analysis of strut-based lattice structures for vibration isolation, Published by 
Precision Engineering, ISSN 0141-6359, Vol. 52, Pp. 494-506.

[4] Aluminium Face Milling. Cutwel [online]. [cit. 2018-03-11].Available

from:https://www.cutwel.co.uk/Aluminium-FaceMilling-at-High-Speed-With-The-Aero-Mill

[5] MIYAGUCHI, T., MASUDA, M., TAKEOKA, E., IWABE, H. (2001) Effect of tool stiffness upon wear in high spindle speed milling using small ball end mill, Published by Precision Engineering, ISSN 0141-6359, Volume 25, Issue 2, Pp. 145154.

[6] WALlACE, P.W., ANDREW, C. (1965) Machining forces. Some effects of tool vibration, $J$ Mech. Eng. Sci. 7, Pp. 152-162.

[7] PETERS, J., VANDEHERCK, P., VAN BRUSSEL, H. (1971) The measurement of the dynamic cutting coefficient, Ann CIRP 21 (2), Pp. 129-136.

[8] TYLER, CH. T., TROUTMAN, J.R., SCHMITZ, T.L. (2016) A coupled dynamics, multiple degree of freedom process damping model, Part 2: Milling, Published by Precision Engineering, ISSN 0141-6359, Vol. 46, Pp. 73-80.

[9] CALVALHO, H.M.B., GOMES, J.O., SCHMIDT, M.A., BRANDAO, V.L.C. (2015) Vibration Analysis and Energy Efficiency in Interrupted Face Milling Processes, Published by Procedia CIRP, ISSN 2212-8271, Vol. 29, Pp. $245-$ 250.

[10] DESHPANDE, V.S., ASHBY, M.F., FLECK, N.A. (2001) Foam topology: bending versus stretching dominated architectures, Published by Acta
Materialia, ISSN 1359-6454, Vol. 49, Issue 6, Pp. 1035-1040.

[11] HAO, L., RAYMONT, D., YAN, C., HUSSIEIN, A., YOUNG, P. Design and Additive Manufacturing of Cellular Lattice Structures, College of Engineering, Mathematics and Physical Sciences, University of Exeter, Exeter EX4 4QF, Devon, United Kingdom.

[12] Damped oscillations. UCSC [online]. [2018-0905]. Available from:https://deutsch.physics.ucsc.edu/6A/book/harmonic/node $18 . h t m l$

[13] Damping. GSU [online]. [2018-09-06]. Available from: http://hyperphysics.phyastr.gsu.edu/hbase/oscda.html\#c2

[14] Harmonic oscillator. Wiki [online]. [2018-09-06]. Available from: https://en.wikipedia.org/wiki/Harmonic_oscillator

[15] WANG, R., SHANG, J., LI, X., LUI, Z., WU, W. (2018) Vibration and damping characteristics of 3D printed Kagome lattice with viscoelastic material filling, Scientific Reports, DOI:10.1038/s41598-018-27963-4.

[16] How to reduce vibration in metal cutting, Sandvik [online]. [cit. 2018-03-11]. Available from: https://www.sandvik.coromant.com/sitecollectiondocuments/downloads/global/technical\%20guides/en-gb/c-2920-23.pdf

[17] BÍLEK, O., PATA, V., KUBIŠOVÁ, M., ŘEZNÍČEK, M. (2018) Mathematical Methods of Surface Roughness Evaluation of Areas with a Distinctive Inclination, Published by Manufacturing Technology, ISSN 1213-2489, June 2018, Vol. 18, No.3, Pp. 363-368. 